\title{
Is cell-based therapy more efficacious for people with amyotrophic lateral sclerosis/motor neuron disease than placebo or no treatment? - A Cochrane review summary with commentary
}

\author{
Maria Gabriella Ceravolo \\ Department of Experimental and Clinical Medicine, Politecnica delle Marche University, Ancona, Italy \\ E-mail:m.g.ceravolo@univpm.it
}

\begin{abstract}
.
BACKGROUND: Even if there is sparse evidence of efficacy of stem cell administration for amyotrophic lateral sclerosis (ALS) in preclinical studies, the clinical use of cell-based therapy is yet to be defined.

OBJECTIVE: To assess the efficacy, feasibility and safety of cell-based therapy in people with ALS/MND, compared with placebo or no treatment.

METHODS: A Cochrane Review on the topic was summarized with comments.

RESULTS: Two randomized controlled trials met the selection criteria, but only one provided data useful for the analysis. It compared autologous bone marrow-mesenchymal stem cells, combined with riluzole, to riluzole only, in 64 people with ALS. The ALS Functional Rating Scale-Revised score slightly improved 6 months after the intervention, though the change was not clinically meaningful. Respiratory function, overall survival and the risk of total adverse events or serious adverse events were not different in the two groups.

CONCLUSIONS: The available evidence does not support the use of bone marrow-mesenchymal stem cells to treat people with ALS/MND.
\end{abstract}

Keywords: Mesenchymal stem cells, amyotrophic lateral sclerosis, functional recovery, respiratory function, ALSFRS-R

The aim of this commentary is to discuss in a rehabilitation perspective the published Cochrane Review "Cell-based therapies for amyotrophic lateral sclerosis/motor neuron disease" (Abdul Wahid et al., 2019) by Abdul Wahid, Law, Ismail, \& $\mathrm{Lai}^{1}$, under the direct

\footnotetext{
${ }^{1}$ This summary is based on a Cochrane Review previously published in the Cochrane Database of Systematic
}

Reviews 2019, Issue 12. Art. No.: CD011742. DOI: 10.1002/ 14651858.CD011742.pub3 (see www.cochranelibrary.com for information). Cochrane Reviews are regularly updated as new evidence emerges and in response to feedback, and Cochrane Database of Systematic Reviews should be consulted for the most recent version of the review. The views expressed in the summary with commentary are those of the Cochrane Corner author and do not represent the Cochrane Library or Wiley. 
supervision of Cochrane Neuromuscular Group. This Cochrane Corner is produced in agreement with $\mathrm{Neu}$ roRehabilitation by Cochrane Rehabilitation.

\section{Background}

Amyotrophic lateral sclerosis (ALS) is an incurable and fatal form of motor neuron disease (MND) presenting with wasting and weakness of all skeletal, respiratory and swallowing muscles (Abdul Wahid et al., 2019). Death usually comes for respiratory failure within three years from symptom onset in $50 \%$ cases. Recent studies support care delivered by a specialist multidisciplinary team as the only strategy associated with improved survival and quality of life (Driskell et al. 2019). The most effective interventions include respiratory support via non-invasive ventilation and nutrition optimization. Timely identification of swallow impairment is the milestone of effective dysphagia management (Andrenelli et al., 2018), while interventions to help patients coping with cognitive changes are yet to be established (NICE, 2016). Etiologic interventions based on stem cell administration have shown promising in preclinical studies, but their clinical efficacy is unclear. This Cochrane Review aims to fill this gap, assessing the evidence now available on cell-based therapy in people with ALS/MND.

\section{CELL-BASED THERAPIES FOR AMYOTROPHIC LATERAL SCLEROSIS/ MOTOR NEURON DISEASE}

(Abdul Wahid, Law, Ismail, \& Lai, 2019)

\section{Objective}

The aim of this Cochrane Review was to assess the efficacy, feasibility and safety of cell-based therapy in people with ALS/MND, compared with placebo or no treatment.

\section{What was studied and methods}

The population addressed in this review was people of any age with a diagnosis of definite or probable ALS/MND according to acknowledged criteria.

Interventions were mononuclear or stem cells, compared with a placebo or no treatment. Cointerven- tions including riluzole and symptomatic treatment were allowed, provided they were administered to both groups equally.

The primary outcome was the Functional impairment, assessed by the ALS Functional Rating Scale-Revised (ALSFRS-R) score at 6 months; secondary outcomes included: a) ALSFRS-R at 12 months, b) Muscle strength, at 6 months, c) Respiratory function (\% predicted forced vital capacity) at 6 months, d) the overall survival at 6 and 12 months, and e) the risk of total adverse events or serious adverse events, at any time-point.

A systematic search of randomized controlled trials (RCTs), quasi-RCTs and cluster RCTs was conducted up to July 31, 2019.

\section{Results}

Out of 151 potentially relevant studies, two RCTs, on a total 112 participants, met the selection criteria. The first trial compared autologous bone marrowmesenchymal stem cells (BM-MSC) administration, combined with riluzole, to riluzole only. The second trial compared the combined intramuscular and intrathecal administration of autologous MSC secreting neurotrophic factors (MSC-NTF) to placebo. Only the first study provided data useful for outcome analysis. They concerned 64 people with ALS (some outcomes available for 56 participants), at 2 years of symptom onset and mild to moderate functional impairment (mean ALSFRS-R score: 35).

The review shows that:

- Functional impairment slightly improved 6 months after treatment with BM-MSC and riluzole compared to riluzole only; the mean difference in ALSFRS-R score was 3.38 points, that is less than the minimum clinically important change (of 4 or more points) (low certainty evidence).

- Respiratory function, overall survival at six months and the risk of total adverse events or serious adverse events were not significantly different in the intervention group, with respect to the control group (all with low certainty evidence).

- Muscle strength was not assessed.

The trial presented a high risk of performance and attrition bias. 


\section{Conclusions}

The authors concluded that current evidence does not support the use of bone marrow-mesenchymal stem cells to treat people with ALS/MND. The only study available for outcome assessment provided low certainty evidence, mainly due to the small sample size. Moreover, it did not address all outcomes of interest. Further RCTs on larger samples and with long-term follow-up are needed to determine the clinical use of cellular therapy and outline the factors that can influence its effectiveness in subjects with ALS/MND. Research should seek to define the best sources, characteristics and methods of administration of stem cells so to design optimal treatment protocols for people with ALS/MND, also including the combination of cell therapy with neuroprotective agents.

\section{Implications for practice in neurorehabilitation}

There is very limited evidence that cell-based therapy provides any benefit to people with ALS/MND. Even if it may slightly reduce motor function decline, it may not improve respiratory function or quality of life or overall survival, at 6 months. Moreover, there is no proof that it may increase muscle strength, or influence swallow and cognitive impairment.

\section{Acknowledgment}

The author thanks Cochrane Rehabilitation and Cochrane Neuromuscular Group for reviewing the contents of the Cochrane Corner.

\section{Conflict of interest}

The author declares no conflicts of interest.

\section{References}

Abdul Wahid S. F., Law Z. K., Ismail N. A., \& Lai N. M. (2019). Cell-based therapies for amyotrophic lateral sclerosis / motor neuron disease. Cochrane Database of Systematic Reviews. Issue 12. Art. No.: CD011742. doi: 10.1002/14651858.CD011742.pub3.

Andrenelli E., Galli F. L., Gesuita R., Skrami E., Logullo F. O., Provinciali L., Capecci M., Ceravolo M. G., \& Coccia M. (2018). Swallowing impairments in amyotrophic lateral sclerosis and myotonic dystrophy type 1: looking for the portrait of dysphagic patient in neuromuscular diseases. NeuroRehabilitation, 42(1), 93-102. doi: 10.3233/NRE-172272

Driskell L. D., York M. K., Heyn P. C., Sanjak M., \& MacAdam C. (2019) A Guide to Understanding the Benefits of a Multidisciplinary Team Approach to Amyotrophic Lateral Sclerosis (ALS) Treatment. Arch Phys Med Rehabil, 100(3), 583-586. doi: 10.1016/j.apmr.2018.05.002

National Institute for Health and Care Excellence. Motor neuron disease: assessment and management. NICE Guideline NG 42. NICE 2016. www.nice.org.uk/guidance/NG42 